\title{
Trastornos del sistema nervioso autónomo Il: pruebas de función autonómica y tratamiento
}

\author{
Autonomic nervous system disorders II: \\ Autonomic function tests and treatment
}

\author{
Alba López-Bravo1,2 \\ 'Sección de Neurología, Hospital Reina Sofía, Tudela, Navarra; \\ 2Instituto de Investigación Sanitaria de Aragón (IIS Aragón), Zaragoza
}

\begin{abstract}
Resumen
El sistema nervioso autónomo (SNA) es una red neuronal compleja que controla diferentes órganos y sistemas. Su evaluación incluye una historia detallada de las funciones autonómicas, examen clínico y pruebas autonómicas. En el pasado, las pruebas de función autonómica no estaban disponibles o eran invasivas. Sin embargo, los avances en la tecnología han mejorado la accesibilidad y disponibilidad de pruebas cuantitativas, no invasivas y reproducibles. No existe un único test para la evaluación de la función autonómica, por lo que se recomienda utilizar una combinación de pruebas que incluyan, entre otras, la evaluación de la función cardiovascular parasimpática y simpática y la función sudomotora. La interpretación de los estudios del SNA es compleja, ya que existe una gran variabilidad interindividual y diferentes factores externos e internos pueden interferir en los resultados. Por ello, se debe llevar a cabo una adecuada preparación del paciente y se requiere una estricta estandarización del procedimiento, utilizando valores y algoritmos predeterminados.
\end{abstract}

Palabras clave: Función autonómica. Sistema nervioso autónomo. Sistema nervioso simpático. Sistema nervioso parasimpático.

\begin{abstract}
The autonomic nervous system (ANS) is a complex neural network that controls several organs and systems. Its assessment includes a detailed history of autonomic functions, clinical examination, and autonomic tests. In the past, methods for ANS evaluation were not available or were invasive. Advances in technology have improved the accessibility and availability of quantitative, non-invasive, and reproducible tests. There is no single test for the evaluation of autonomic function, for this, it is recommended to use a combination of tests that include the evaluation of parasympathetic and sympathetic cardiovascular function and sudomotor function. The interpretation of the test results is often difficult due to the complexity of the individual reflex arcs and the fact that many external and internal disturbances can influence the test results. This requires strict standardization of patient preparation, test procedures and evaluation using standard values and test algorithms created for all laboratories.
\end{abstract}

Keywords: Autonomic function testing. Autonomic nervous system. Sympathetic nervous system. Parasympathetic nervous system.

\section{Autor de correspondencia:}

Alba López-Bravo

E-mail: alba.lopez.bravo@gmail.com
Fecha de recepción: 29-10-2021

Fecha de aceptación: 07-11-2021

DOI: 10.24875/KRANION.M21000007
Disponible en internet: 24-12-2021

Kranion. $2021 ; 16: 79-84$

www.kranion.es

1577-8843 / () 2021 Kranion. Publicado por Permanyer. Este es un artículo open access bajo la licencia CC BY-NC-ND (http://creativecommons.org/licenses/by-nc-nd/4.0/). 


\section{INTRODUCCIÓN}

El sistema nervioso autónomo (SNA) está constituido por el sistema nervioso simpático, el sistema nervioso parasimpático y el sistema nervioso entérico. El SNA constituye una parte importante del sistema nervioso y regula funciones tan importantes como: presión arterial $(P A)$, frecuencia cardíaca (FC), termorregulación, respiración, y sistemas gastrointestinal, urogenital y pupilar.

Las manifestaciones clínicas del SNA son diversas y por ello es necesaria una evaluación clínica de amplio alcance. Las pruebas de función autonómica son una extensión de la historia clínica y del examen físico y deben ser efectuadas por un médico experto que interprete de forma conjunta los hallazgos encontrados. Pese a que la mayoría de ellas son fáciles de realizar, su interpretación es compleja y diferentes factores externos e internos pueden interferir en los resultados. Por ello, se requiere una estricta estandarización del procedimiento, utilizando valores y algoritmos predeterminados y se debe llevar a cabo una adecuada preparación del paciente antes de su realización (Tabla 1).

El compromiso del SNA puede presentarse en enfermedades del sistema nervioso central, del sistema nervioso periférico y en patologías primarias del SNA. En otros casos, la afectación del SNA puede ser funcional, sin evidencia de una lesión estructural de las vías autonómicas. Las principales indicaciones clínicas para realizar un estudio de función autonómica son las siguientes ${ }^{1}$ :

- Sospecha de insuficiencia autónoma generalizada. Puede deberse a la presencia de fallo autonómico puro (FAP), atrofia multisistémica (AMS) o neuropatías autonómicas (p. ej., diabética, amiloidótica, etc.).

- Sospecha de intolerancia ortostática (IO). La IO, incluida la hipotensión ortostática, el síncope o el síndrome de taquicardia ortostática postural (POTS, postural orthostatic tachycardia syndrome) suponen la presencia de síntomas de hipoperfusión cerebral o hiperactividad autónoma al adoptar el ortostatismo, con resolución en la posición de decúbito. En este caso, las pruebas autonómicas cardiovasculares nos ayudarán a evaluar la presencia de 10 y su gravedad.

- Diagnóstico de trastornos autonómicos benignos. Por ejemplo, la anhidrosis idiopática crónica, en la que los estudios de función simpática y parasimpática son normales.

- Diagnóstico diferencial. Ayuda a determinar la gravedad y a diferenciar entre distintas etiologías como la enfermedad de Parkinson (EP), la AMS y la FAP.

- Monitorización de la evolución clínica en pacientes con disfunción autonómica.

- Evaluación de la respuesta al tratamiento en pacientes con disfunción autonómica.

En la actualidad no existe un único test para la evaluación de la función autonómica, así que con el objetivo
Tabla 1. Preparación del paciente antes del estudio de función autonómica

Aproximadamente 48 horas antes se debe suspender

lo siguiente:

- Anticolinérgicos (p. ej., antihistamínicos, antidepresivos)

- Simpaticomiméticos ( $\alpha$-agonistas y $\beta$-agonistas)

- Parasimpaticomiméticos

- Mineralocorticoides (p. ej., 9- $\alpha$-fludrocortisona)

- Diuréticos

Aproximadamente 24 horas antes se debe suspender

lo siguiente:

- Simpaticolíticos ( $\alpha$-antagonistas y $\beta$-antagonistas)

Aproximadamente 12 horas antes se debe suspender lo siguiente:

- Alcohol

- Analgésicos

En la mañana del estudio:

- No utilizar ropa ajustada

- No utilizar corsé

- No utilizar medias de compresión

Aproximadamente 3 horas antes se debe suspender

lo siguiente:

- Nicotina

- Cafeína

- Comida

Adaptada de Ziemssen, et al., 2019'.

de sintetizar algunas de las pruebas disponibles proponemos el esquema por aparatos que se desarrolla a continuación.

\section{EVALUACIÓN DEL APARATO CARDIOVASCULAR}

\section{Pruebas ortostáticas}

La hipotensión ortostática $(\mathrm{HO})$ es la incapacidad para adaptarse al ortostatismo, lo que resulta en una caída progresiva de la PA que ocurre hasta que aparecen síntomas. La prueba ortostática puede llevarse a cabo de forma activa al adoptar la bipedestación o pasiva mediante el test de la mesa basculante. Se coloca al paciente en una mesa motorizada, contenido mediante un cinturón y permanece unos 15 minutos en decúbito supino. Posteriormente, se inclina la mesa hasta un ángulo de 60 $80^{\circ}$ y se mantiene esta posición 40-60 minutos o hasta que se desencadenan síntomas de $\mathrm{HO}^{2}$. En condiciones normales, tras la bipedestación, la PA sistólica desciende unos $10 \mathrm{mmHg}$, mientras que la diastólica aumenta unos $5 \mathrm{mmHg}$. De la misma forma, la FC aumenta entre 5 y 20 latidos por minuto. La HO se define como una caída 
de la PA sistólica igual o superior a 20 o de $10 \mathrm{mmHg}$ de la PA diastólica en los primeros tres minutos tras la bipedestación 0 inclinación en la mesa basculante ${ }^{3}$.

\section{Masaje del seno carotídeo}

Debe utilizarse en aquellos pacientes en los que se sospecha de una hipersensibilidad del seno carotídeo. Con el paciente acostado y bajo monitorización de PA y electrocardiográfica, se palpa y ejerce una ligera presión en el seno carotídeo durante 20-30 segundos de forma secuencial. La maniobra conduce fisiológicamente a una reducción moderada de la FC y de la PA. Se considera que la prueba es positiva si tras 2 minutos se alcanza una reducción de la PA sistólica de más de 50 mmHg o se consigue una pausa ventricular superior a 3 segundos 4 . Debe evitarse en pacientes con patología carotídea o antecedente reciente de ictus.

\section{Respuesta de la frecuencia cardíaca tras la respiración profunda}

Es la prueba más utilizada para la evaluación del sistema nervioso parasimpático. En condiciones fisiológicas, la FC se incrementa en respuesta a la inspiración y disminuye con la espiración. Para obtener información sobre la variabilidad de la FC, se pide al paciente que respire profunda y uniformemente (inspiración 5 segundos y espiración 7 segundos) durante un minuto y bajo monitorización electrocardiográfica. En condiciones normales, el intervalo más largo entre latidos (R-R) durante la espiración es al menos 1,15 veces mayor que el mínimo intervalo R-R durante la inspiración ${ }^{5}$.

\section{Respuesta de la frecuencia cardíaca tras el cambio postural}

Los cambios de FC en los primeros 30 segundos tras adoptar la bipedestación permiten una evaluación del sistema parasimpático cardíaco. La proporción 30:15 o «proporción de Ewing» mide la relación entre la FC a los 15 segundos frente a la relativa bradicardia que se produce a los 30 segundos. La proporción 30:15 representa un índice reproducible dependiente de la función cardiovagal y fisiológicamente tiene un valor $>1,04^{6}$.

\section{Respuesta de la frecuencia cardíaca tras la realización de maniobras de Valsalva}

Los cambios en la FC durante la maniobra de Valsalva se consideran una medida sensible y específica de la función parasimpática. Tras una inspiración profunda, se pide al paciente que realice una exhalación a través de un tubo conectado a un manómetro hasta alcanzar 30-50 mmHg durante 10-15 segundos. Después de un breve aumento de la PA debido a la compresión mecá- nica de la aorta (fase I), se produce una reducción de la precarga cardíaca y del volumen sistólico con caída de la PA (fase II temprana). Posteriormente, la activación de los barorreceptores provoca una activación simpática con elevación de la PA (fase II tardía) y al completarse el aumento de la presión intratorácica se produce un aumento excesivo de la PA diastólica y sistólica (fase III). Al finalizar la maniobra tiene lugar un incremento máximo de la PA por recuperación de la eyección sistólica y activación simpática (fase IV) y como consecuencia de la actividad del reflejo barorreceptor se produce una bradicardia posterior. En condiciones normales se produce un aumento $\geq 10 \mathrm{mmHg}$ entre la PA diastólica final y la PA diastólica inicial. Por otra parte, la proporción entre el intervalo R-R máximo después de la maniobra y el intervalo R-R mínimo durante la maniobra debe ser superior a $1,20^{7}$.

\section{Pruebas de imagen de medicina nuclear}

La función simpática miocárdica puede ser evaluada mediante técnicas de gammagrafía cardíaca, que utiliza ${ }^{123}$ |-metayodobencilguanidina (123/-MIBG). El ${ }^{123}$ |-MIBG es un análogo de la norepinefrina (NE) y su utilización permite valorar la distribución e integridad de la inervación simpática posganglionar. Tras la administración del radiofármaco, se evalúan los niveles de captación de radiotrazador a los 15 minutos y su tasa de lavado en las 4 horas posteriores. Por otra parte, la exploración mediante tomografía por emisión de protones tras la administración sistémica de agentes como 11C-hidroxiefedrina, 11C-EPI o 6-fluorodopamina, permite una rápida visualización de la inervación simpática cardíaca con excelente resolución espacial. Sin embargo, debido a su menor disponibilidad, se utiliza con menor frecuencia.

\section{EVALUACIÓN DE LA RESPUESTA TERMORREGULADORA Y SUDOMOTORA}

\section{Test cuantitativo del reflejo axónico sudomotor}

El test cuantitativo del reflejo axónico sudomotor (QSART) se emplea para evaluar la integridad funcional del axón sudomotor simpático posganglionar. Consiste en una estimulación eléctrica en la piel mediante iontoforesis de acetilcolina al 10\% para activar las glándulas sudoríparas. EI QSART mide el volumen de sudor producido tras esta estimulación por medio de un higrómetro ${ }^{8}$.

\section{Test de sudoración termorreguladora}

El test de sudoración termorreguladora (TST) evalúa la integridad de las vías sudomotoras simpáticas y establece un diagnóstico topográfico de las áreas corporales con un trastorno de la sudoración, sin embargo, no permite 
establecer el nivel lesional. Se coloca al paciente en decúbito en una cámara con temperatura $\left(45-50^{\circ} \mathrm{C}\right)$ y humedad (35-40\%) controladas. Previamente, el paciente ha sido impregnado con polvo indicador (rojo de alizarina o quinizarina) que cambia de color con los cambios en el pH producidos por la sudoración. Se procede al aumento de temperatura de la habitación hasta conseguir un incremento en la temperatura oral de $1^{\circ} \mathrm{C}$ o bien alcanzar los $38^{\circ} \mathrm{C}$, mientras se toman imágenes que permiten crear un mapa digital de densidad de sudor. Finalmente, los resultados se expresan en porcentaje, en función del área de anhidrosis en el total de la superficie corporal (Fig. 1)9.

\section{Respuesta simpática cutánea}

Es útil para evaluar la integridad de la función colinérgica simpática periférica al medir los cambios de la actividad eléctrica de la piel provocados por diferentes estímulos. El paciente debe permanecer en reposo, en decúbito supino; se coloca el electrodo activo en la palma o planta y el de referencia en la región dorsal y se estimula el nervio mediano o tibial contralateral, midiendo la amplitud y latencia de la respuesta mediante electromiografía. El resultado se considera anormal si existe una diferencia de al menos el 50\% entre las amplitudes de ambos lados o si la respuesta está ausente. Esta respuesta resulta de la activación de la vía eferente simpática desde el hipotálamo hasta la inervación colinérgica periférica, sin embargo, no permite establecer el nivel lesional y existen variaciones interindividuales e intraindividuales que limitan su utilidad ${ }^{10}$.

\section{EVALUACIÓN DE LA RESPUESTA OCULOMOTORA}

\section{Test pupilares con colirios}

Cuando se produce denervación pupilar, existe una hipersensibilidad a la instilación de agentes parasimpaticomiméticos. Para evidenciar el tipo y grado de denervación, se utilizan test pupilares con colirios como la pilocarpina y la hidroxianfetamina. Si existe un trastorno parasimpático, la utilización de pilocarpina al 1\% provocará miosis pupilar debido a una hipersensibilidad por denervación del músculo del iris. La hidroxianfetamina es un agente simpaticomimético que permite evaluar la integridad de las funciones adrenérgicas simpáticas de la pupila y localizar el lugar de la lesión, de forma que provoca dilatación pupilar en el caso de pupilas normales y lesiones preganglionares, pero no en el caso de lesiones posganglionares ${ }^{11}$.

\section{Pupilometría}

Se basa en la medición de los diámetros pupilares y permite valorar de forma cuantitativa la integridad de la actividad neuronal a lo largo de las vías visuales ${ }^{12}$. El paciente debe permanecer sentado en un cuarto oscuro, con

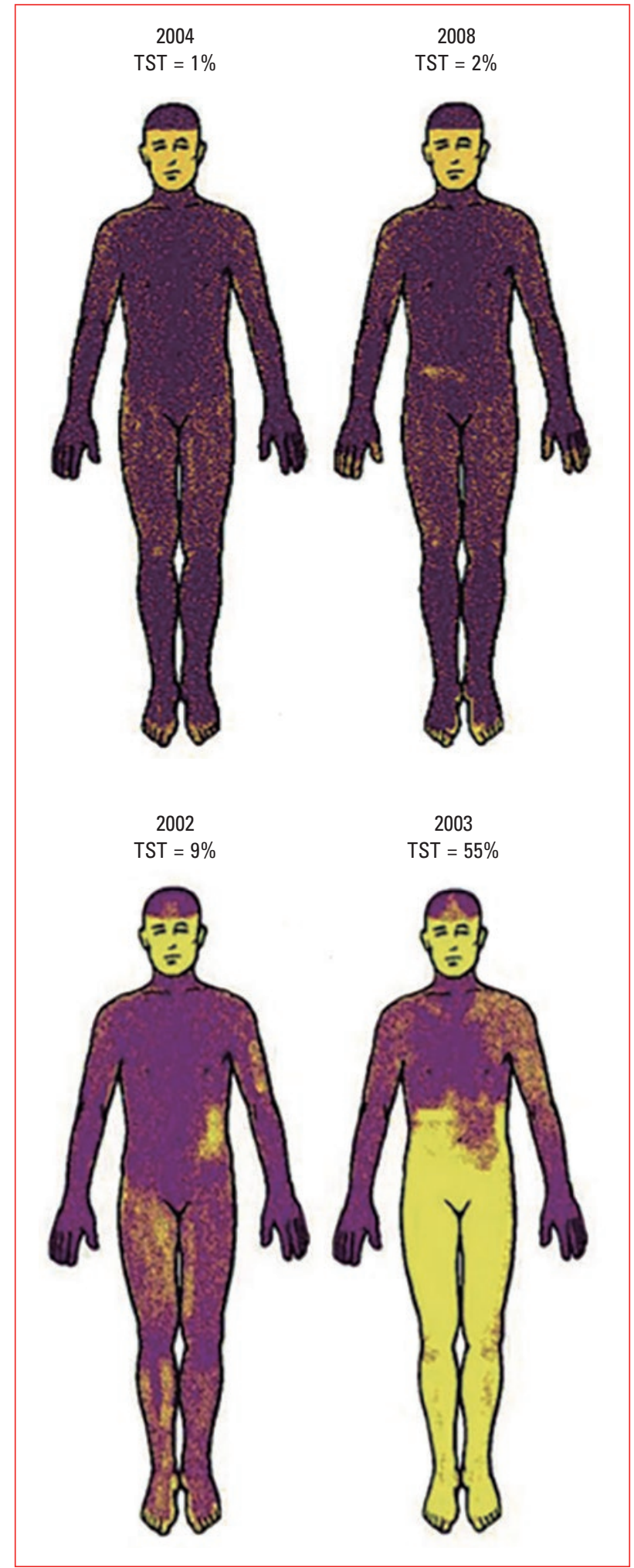

Figura 1. Test de sudoración termorreguladora (TST). En la figura aparecen los patrones característicos de las sinucleinopatías en el TST. En la imagen superior se muestra el patrón de anhidrosis distal en un paciente con enfermedad de Parkinson. En el panel inferior se observa el patrón característico de una paciente con atrofia multisistémica, con anhidrosis regional y un porcentaje mayor y con progresión más rápida en el tiempo (adaptada de Low, et al., 20134). 
TABLA 2. Medidas no farmacológicas para el tratamiento de la hipotensión ortostática

\begin{tabular}{|c|c|c|}
\hline Medida & Mecanismo de acción & Recomendación \\
\hline $\begin{array}{l}\text { Evitar comidas copiosas y abundantes } \\
\text { en hidratos de carbono }\end{array}$ & $\downarrow$ la vasodilatación esplácnica & Ingerir cantidades pequeñas y frecuentes de comida \\
\hline Realizar actividad física & $\uparrow$ la masa muscular & $\begin{array}{l}\text { Realizar ejercicio en decúbito supino } \\
\text { Evitar el reposo prolongado }\end{array}$ \\
\hline $\begin{array}{l}\text { Mantener una buena hidratación } \\
\text { e ingesta de sal }\end{array}$ & $\begin{array}{l}\uparrow \text { el volumen intravascular } \\
\downarrow \text { la producción de óxido nítrico }\end{array}$ & Ingerir de 2 a 2,5 I de líquidos y de 10 a $20 \mathrm{~g}$ de sal/día \\
\hline Realizar maniobras de contrapresión & $\uparrow$ la PA y el gasto cardíaco & $\begin{array}{l}\text { Cruzar las piernas, inclinarse hacia adelante, ponerse } \\
\text { en "cuclillas" }\end{array}$ \\
\hline Utilizar prendas de compresión & $\uparrow$ la PA & $\begin{array}{l}\text { Compresión abdominal }(20-30 \mathrm{mmHg}) \text { y hasta muslo } \\
(30-40 \mathrm{mmHg})\end{array}$ \\
\hline Elevar la cabecera de la cama & $\downarrow$ la diuresis nocturna & Elevar la cabecera de la cama 12 grados \\
\hline
\end{tabular}

PA: presión arterial. Adaptada de Chelimsky, et al., $2020^{15}$.

los ojos abiertos y la cabeza firme, fijando su mirada en un punto lejano frente a él. Mediante una cámara infrarroja, se evalúan el tiempo promedio de latencia de constricción, el tiempo promedio de latencia de constricción de la velocidad máxima y el tiempo de latencia de la dilatación. Una diferencia mayor de $1 \mathrm{~mm}$ de diámetro entre los ojos, un porcentaje de cambio pupilar antes/después de la estimulación menor al 10\% y una velocidad de constricción menor de $0,8 \mathrm{~mm} / \mathrm{s}$ se consideran valores anormales ${ }^{13}$.

\section{EVALUACIÓN DEL APARATO DIGESTIVO}

\section{Videofluoroscopia}

Es una técnica útil para la valoración de la deglución y la presencia de disfagia orofaríngea. Permite además apreciar en tiempo real imágenes del bolo alimenticio en relación con el movimiento estructural durante la deglución y el riesgo de broncoaspiración ${ }^{13}$.

\section{Manometría esofágica}

Valora la presencia y el grado de integridad de la peristalsis mediante la determinación de las presiones del esfínter esofágico inferior y los músculos del esófago en diferentes fases.

\section{EVALUACIÓN DEL APARATO URINARIO}

\section{Ecografía vesical con medición del residuo posmiccional}

Es una prueba sencilla, barata y no invasiva, que puede ser realizada en la práctica clínica. Un volumen urinario residual $\geq 100 \mathrm{ml}$ (normal $<50 \mathrm{ml}$ ) es indicativo de una vejiga hipoactiva.

\section{Estudios urodinámicos invasivos}

Los estudios urodinámicos evalúan las funciones simpática (relajación de los músculos de la vejiga, contracción del músculo liso uretral) y parasimpática (contracción de la pared de la vejiga, relajación del esfínter interno) del sistema urinario. Existen diferentes pruebas que evalúan la integridad de la función de la vejiga y el esfínter: flujometría (volumen de almacenamiento y velocidad de vaciado de la vejiga), cistometría (función de llenado y vaciado de la vejiga) y medición del perfil uretral o perfilometría (registro del caudal y presión necesaria para orinar).

\section{EVALUACIÓN DE LA FUNCIÓN SEXUAL}

\section{Pletismografía del pene}

Valora la función sexual parasimpática analizando los cambios de volumen y flujo sanguíneo en el pene para determinar la integridad de la erección masculina.

\section{Test de papaverina}

La inyección intracavernosa de papaverina evalúa la actividad parasimpática de la función eréctil masculina. Tras su administración, se debe alcanzar una erección completa dentro de los 10 a 15 minutos posteriores a la inyección, con una duración de más de 10 a 30 minutos $^{14}$.

\section{TRATAMIENTO DE LOS TRASTORNOS DEL SISTEMA NERVIOSO AUTÓNOMO}

Un principio fundamental al considerar el tratamiento de los trastornos del SNA es que si existe una causa tratable que subyace a la disfunción autónoma (p. ej., neuropatía inflamatoria aguda, diabética o tóxica) se debe llevar a cabo el tratamiento etiológico. 
TABLA 3. Tratamientos farmacológicos utilizados en la hipotensión ortostática

\begin{tabular}{|c|c|c|c|c|}
\hline Tratamiento & Fludrocortisona & Piridostigmina & Midodrina & Droxidopa \\
\hline \multirow[t]{2}{*}{$\begin{array}{r}\text { Mecanismo } \\
\text { de acción }\end{array}$} & Mineralocorticoide & Inhibidor de acetilcolina & $\begin{array}{l}\text { Agonista selectivo de los } \\
\text { receptores } \alpha 1 \text { adrenérgicos }\end{array}$ & $\begin{array}{l}\text { Precursor de } \\
\text { noradrenalina }\end{array}$ \\
\hline & $\begin{array}{l}\uparrow \text { PA al aumentar el } \\
\text { volumen intravascular }\end{array}$ & $\begin{array}{l}\text { Mejora la transmisión } \\
\text { ganglionar }\end{array}$ & $\begin{array}{l}\uparrow \text { PA al aumentar la } \\
\text { resistencia vascular }\end{array}$ & $\begin{array}{l}\uparrow \text { PA al inducir } \\
\text { vasoconstricción }\end{array}$ \\
\hline Dosis & $\begin{array}{r}0,1-0,2 \mathrm{mg} / 24 \mathrm{~h} \\
(2 \text { tomas/día) }\end{array}$ & $\begin{array}{l}30-60 \mathrm{mg} / 24 \mathrm{~h} \\
\quad(1-3 \text { tomas/día) }\end{array}$ & $\begin{array}{l}\text { Inicio con } 2,5 \mathrm{mg} \\
\text { e incremento progresivo } \\
\text { hasta } 30 \mathrm{mg} / 24 \mathrm{~h} \\
\text { Última dosis }>4 \mathrm{~h} \text { antes } \\
\text { de acostarse }\end{array}$ & $100-600 \mathrm{mg} / 24 \mathrm{~h}$ \\
\hline $\begin{array}{l}\text { Efectos } \\
\text { adversos }\end{array}$ & $\begin{array}{l}\text { Aumento de peso } \\
\text { Hipopotasemia } \\
\text { Descompensación } \\
\text { cardiaca }\end{array}$ & $\begin{array}{l}\text { Síntomas gastrointestinales } \\
\text { Aumento de salivación } \\
\text { y sudoración } \\
\text { Incontinencia urinaria }\end{array}$ & $\begin{array}{l}\text { Hipertensión arterial } \\
\text { en decúbito } \\
\text { Parestesias } \\
\text { Prurito }\end{array}$ & $\begin{array}{l}\text { Cefalea } \\
\text { Mareo } \\
\text { Náuseas } \\
\text { Hipertensión arterial }\end{array}$ \\
\hline
\end{tabular}

PA: presión arterial. Adaptada de Isaacson, et al., 202176.

Una de las manifestaciones más frecuentes de la disfunción autónoma es la HO, presente en pacientes con trastornos neurodegenerativos como la AMS, EP, FAP y neuropatías autonómicas. El objetivo del tratamiento es disminuir la incidencia y gravedad de los síntomas posturales y permitir que el paciente pueda estar de pie durante periodos de tiempo más largos para poder realizar sus actividades de forma independiente. El manejo inicial de la $\mathrm{HO}$ se basa en proporcionar al paciente consejos sobre el estilo de vida y la utilización de medidas no farmacológicas ${ }^{15}$ (Tabla 2).

El tratamiento farmacológico de la $\mathrm{HO}$ se basa en la expansión de volumen y la vasoconstricción. Los fármacos más utilizados para su tratamiento son: fludrocortisona, piridostigmina, midodrina y droxidopa ${ }^{16}$ (Tabla 3). En aquellos pacientes en los que el tratamiento con fludrocortisona es ineficaz, puede valorarse terapia adyuvante con efedrina, indometacina, propranolol, atenolol o clonidina. En algunos casos de $\mathrm{HO}$ grave se han utilizado marcapasos atriales e infusiones intravenosas intermitentes de norepinefrina, siendo necesaria en estos casos una evaluación adicional.

\section{FINANCIACIÓN}

Ninguna.

\section{CONFLICTOS DE INTERESES}

Ninguno.

\section{RESPONSABILIDADES ÉTICAS}

\section{Protección de personas y animales}

Los autores declaran que para esta investigación no se han realizado experimentos en seres humanos ni en animales.

\section{Confidencialidad de los datos}

Los autores declaran que en este artículo no aparecen datos de pacientes.

\section{Derecho a la privacidad y consentimiento informado}

Los autores declaran que en este artículo no aparecen datos de pacientes.

\section{BIBLIOGRAFÍA}

1. Ziemssen T, Siepmann T. The Investigation of the cardiovascular and sudomotor autonomic nervous system-A review. Front Neurol. 2019;10:53.

2. Aydin AE, Soysal P, Isik AT. Which is preferable for orthostatic hypotension diagnosis in older adults: active standing test or head-up tilt table test? Clin Interv Aging. 2017;12:207-12

3. Freeman R, Wieling W, Axelrod FB, Benditt DG, Benarroch E, Biaggioni I, et al. Consensus statement on the definition of orthostatic hypotension, neurally mediated syncope and the postural tachycardia syndrome. Clin Auton Res. 2011; 21(2):69-72.

4. Low PA, Tomalia VA, Park KJ. Autonomic function tests: some clinical applications. J Clin Neurol. 2013;9(1):1-8

5. Zaza A, Lombardi F. Autonomic indexes based on the analysis of heart rate variability: a view from the sinus node. Cardiovasc Res. 2001;50(3):434-42.

6. Ewing DJ, Campbell IW, Clarke BF. Heart-rate response to standing as a test for automatic neuropathy. Br Med J. 1978;1(6128):1700.

7. Hilz MJ, Dütsch M. Quantitative studies of autonomic function. Muscle Nerve. 2006;33(1):6-20.

8. Low PA, Denq JC, Opfer-Gehrking TL, Dyck PJ, O'Brien PC, Slezak JM. Effect of age and gender on sudomotor and cardiovagal function and blood pressure response to tilt in normal subjects. Muscle Nerve. 1997;20:1561-8.

9. Low PA. Evaluation of sudomotor function. Clin Neurophysiol. 2004;115(7): 1506-13.

10. Vetrugno R, Liguori R, Cortelli P, Montagna P. Sympathetic skin response: basic mechanisms and clinical applications. Clin Auton Res. 2003;13(4):256-70.

11. Ravits JM. AAEM minimonograph \#48: autonomic nervous system testing. Muscle Nerve. 1997;20:919-37

12. León-Sarmiento FE, Prada DG, Gutiérrez C. Pupilas, pupilometría y pupilografía. Acta Neurol Colomb. 2008;24:188-97.

13. Quispe RC, Novak P. Auxiliary tests of autonomic functions. J Clin Neurophysiol. 2021;38(4):262-73.

14. Elhanbly S, Schoor R, Elmogy M, Ross L, Hegazy A, Niederberger C. What nonresponse to intracavernous injection really indicates: a determination by quantitative analysis. J Urol. 2002;167:192-6.

15. Chelimsky G, Chelimsky T. Non-pharmacologic management of orthostatic hypotension. Auton Neurosci. 2020;229:102732.

16. Isaacson SH, Dashtipour K, Mehdirad AA, Peltier AC. Management strategies for comorbid supine hypertension in patients with neurogenic orthostatic hypotension. Curr Neurol Neurosci Rep. 2021;21(4):18. 